\title{
SIMULAÇÃO DA INFLUÊNCIA DO EQUILÍBRIO DE FASES DO TERNÁRIO ÁGUA-ETANOL-DEE NO PROCESSO DE PRODUÇÃO DO DIETIL ÉTER POR DESIDRATAÇÃO DO ETANOL
}

\author{
R. M. NICODEMOS ${ }^{1}$, F. RODRIGUES ${ }^{1}$ e A. J. DE ASSIS ${ }^{1}$ \\ ${ }^{1}$ Universidade Federal de Uberlândia - Faculdade de Engenharia Química - Núcleo de Modelagem, \\ Controle e Otimização de Processos \\ E-mail: renata.mnicodemos@gmail.com
}

\begin{abstract}
RESUMO- Para os simuladores de processos, a escolha dos pacotes termodinâmicos é dificultada pela ausência de bancos de dados completos e dados experimentais disponíveis na literatura, principalmente para misturas ternárias. A proposta deste trabalho é apontar o melhor modelo termodinâmico para a simulação de uma planta de produção de dietil éter (DEE) por meio da desidratação do etanol. Para isto, os resultados obtidos foram validados com dados experimentais, assim como foi analisada a variação das cargas térmicas dos equipamentos e a volatilidade relativa das colunas de destilação presentes na planta simulada. Os simuladores utilizados foram o COCO e o ChemSep. O melhor modelo para o ELV do binário água-etanol e para o ELL ternário água-etanol-DEE foi o UNIQUAC. Assim, ao unir num mesmo trabalho a validação de pacotes termodinâmicos, simulação e impacto da escolha dos pacotes termofísicos em parâmetros-chave, espera-se contribuir para a área da Engenharia dos Processos Químicos.
\end{abstract}

\section{INTRODUÇÃO}

O uso de simuladores de processos, antes restrito a apenas algumas áreas, está cada vez mais presente tanto na área acadêmica quanto no setor industrial. Os simuladores de processos podem ser utilizados com ferramenta de apoio para a síntese de processos químicos, poupando tanto dinheiro quanto tempo, e a otimização de plantas já em operação. A única restrição ao seu uso é a limitação dos bancos de dados termofísicosdisponíveis nos simuladores de processos assim como as diferenças de resultados que são encontrados de um simulador para outro (SADEQ et al, 1997).

Os bancos de dados das propriedades termofísicas são baseados em equações empíricas, equações de estado, modelos de energia, etc. A dificuldade em ter estes bancos de dados completos é proveniente da falta de dados experimentais disponíveis, ou por serem difíceis de coletar ou pelo custo. Isto impossibilita o cálculo dos parâmetros dos modelos que utilizam dados experimentais como referência em outras temperaturas e pressões.

A idéia principal da simulação de processos é a de que a simulação seja fidedigna ao processo real e para que isto seja possível a escolha do pacote termodinâmico que calculará as propriedades termofísicas deve ser feita com embasamento teórico, ou seja, validando os resultados obtidos no simulador de processos com os dados físicos da planta real ou com dados experimentais das propriedades isoladas.

Para comprovar como a escolha do pacote termodinâmico interfere nos resultados da simulação, foi escolhida uma planta de produção de dietil éter (DEE) por meio da desidratação do etanolpara fazer a análise comparativa. O objetivo é verificar as diferenças nos resultados encontrados quando os pacotes termodinâmicos são alterados nos equilíbrios de fase. Todos os resultados obtidos no simulador de processo foram comparados com dados experimentais encontrados na literatura. Também foram comparados os valores das cargas térmicas das colunas de 
destilação e do reator para cada modelo analisado e o comportamento da volatilidade relativa dos componentes nas colunas de destilação.

\section{METODOLOGIA}

\subsection{SOFTWARES UTILIZADOS}

\section{COCO (CAPE OPEN TO CAPE OPEN)}

As simulações realizadas neste trabalho foram feitas com o simulador de processos COCO (Cape Open to Cape Open), que é um simulador gratuito de processos no estado estacionário. O simulador COCO é dividido em quatro sub-aplicativos:

- COFE - é a interface gráfica para o usuário; possuiu um algoritmo de solução sequencial e exibe os dados das correntes e dos equipamentos.

- TEA - é o pacote de dados termodinâmicos baseado na biblioteca do ChemSep incluindo aproximadamente 430 substâncias e mais de 100 métodos de cálculo de propriedades.

- COUSCOUS - é o pacote que engloba as operações unitárias disponíveis no simulador.

- CORN - pacote de reações químicas; a partir deste pacote é possível especificar o tipo de cinética ou uma reação de equilíbrio.

\section{Chem-Sep LITE}

O softareChem-Sep LITE é instalado ao computador juntamente com a instalação do simulador de processos COCO. Trata-se de uma simulador para colunas de destilação, adsorção e operações de extração.

\section{Veusz}

É um aplicativo em que é possível plotar gráficos científicos como: gráficos ternários, gráficos polares, etc. É possível inserir, manipular e examinar dados dentro do aplicativo. O programa roda tanto no Windows quanto no Unix/Linux e Mac OS X.

\subsection{ESTUDO DE CASO: PLANTA INDUSTRIAL DE PRODUÇÃO DE DEE E SEPARAÇÃO DO TERNÁRIO ÁGUA/ETANOL/DEE}

Dietil éter pode ser produzido tanto por catálise homogênea quanto por catálise heterogênea. A catálise homogênea tem comportamento corrosivo, já que utiliza ácido sulfúrico no processo, o que aumenta o custo do processo. No geral, a desidratação do etanol para formar o dietil éter é feita a altas temperaturas (WIDAYAT et al., 2013). Na desidratação do etanol à temperaturas inferiores a $260^{\circ} \mathrm{C}$, a produção é de dietil éter é quase única. Para temperaturas acima de $300^{\circ} \mathrm{C}$, a reação gera etileno e água.

A Figura 1 mostra o fluxograma da simulação no simulador de processos COCO. A simulação é constituída por duas colunas de destilação sendo uma responsável pela separação do DEE da mistura binária água/etanol (ChemSepUO_2) e a outra coluna de destilação responsável pela separação do binário água/etanol (ChemSepUO_3). O fluxograma apresenta um reator de conversão fixa a $40^{\circ} \mathrm{C}$ que desidrata $50 \%$ do etanol em dietil éter (DEE) e água. 


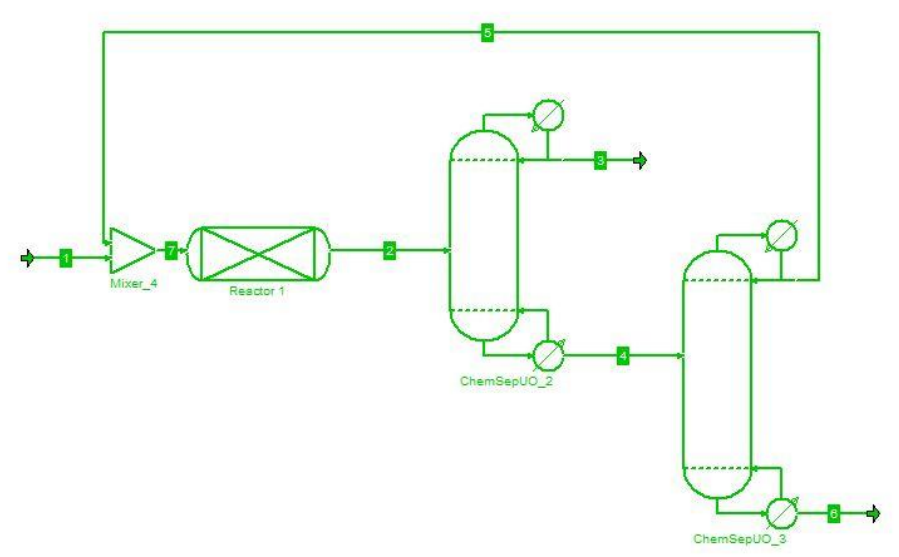

Figura 1- Fluxograma da simulação realizadano COCO para a planta de desidratação do etanol

\section{RESULTADOS}

A simulação foi realizada utilizando o modelo UNIFAC para o cálculo do coeficiente de atividade e atividade da fase líquida e a equação de estado usada para a fase vapor foi a Lei dos Gases Ideais. Como a simulação inteira ocorre a pressão de $1 \mathrm{~atm}$ (pressão baixa), não há necessidade de avaliar o efeito da escolha da equação de estado para a fase vapor, já que a variação de resultados é pequena.Para a validação do ELV para o binário água-etanol foram usados quatro modelos: UNIFAC, NRTL, UNIQUAC e Wilson. O resultado estão mostrados na Figura 2.

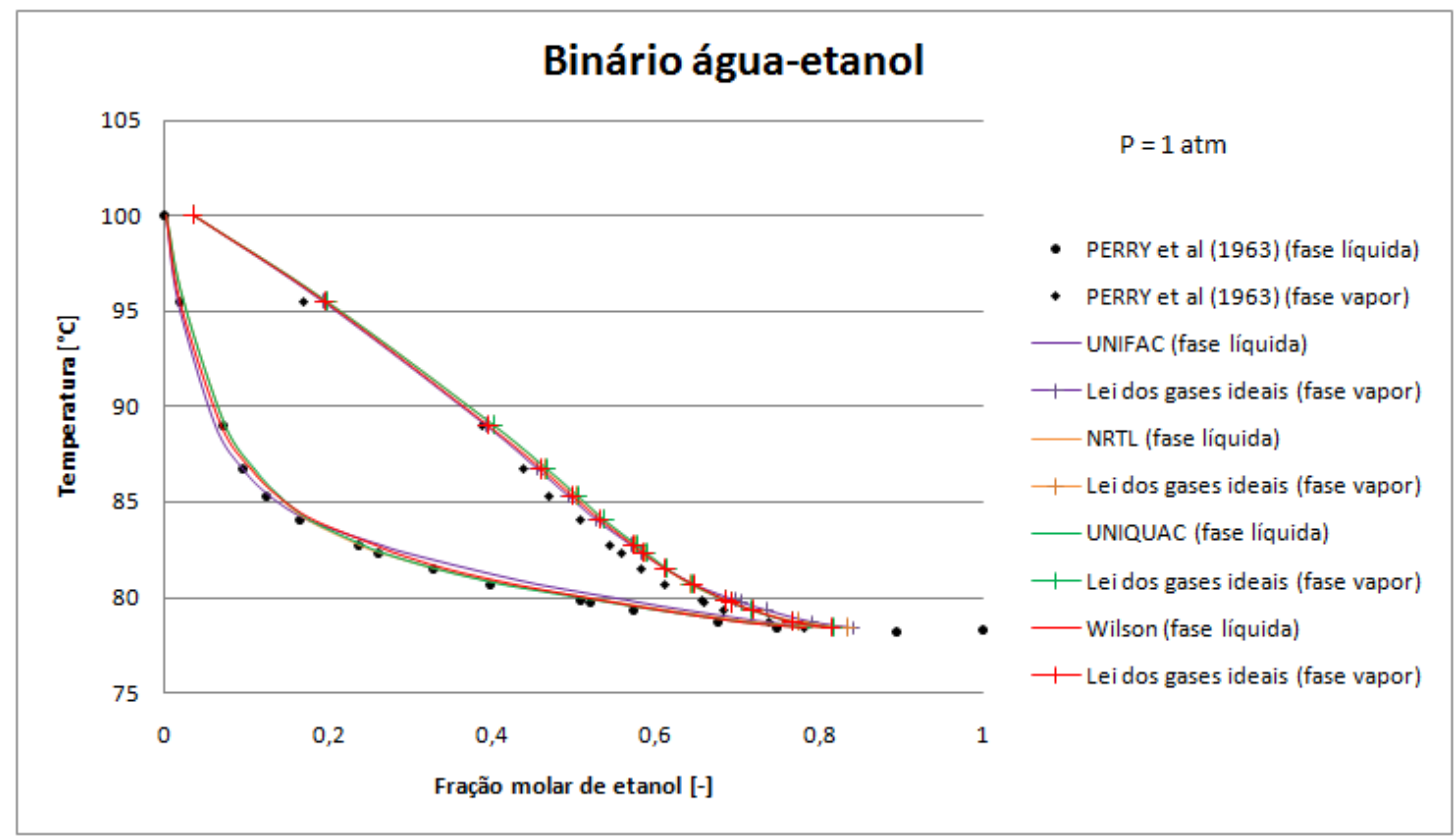

Figura 2- Diagramas ELV a pressão constante comparando dados experimentais e modelos para a mistura binária água-etanol

Apenas com a análise da Figura 2 não é possível verificar qual é o modelo que melhor descreve o binário água-etanol, para isto foi calculado o desvio padrão médio entre os dados experimentais (PERRY et al.,1963) e os dados simulados. O resultado está presente na Tabela 1.

O menor erro relativo médio para a fase líquida (fase que está sendo analisada) é de 12,97\% para o modelo UNIQUAC. O modelo de coeficiente de atividade UNIQUAC é adequado para prever atividade de sistemas binário do tipo água/orgânicos (gás ideal/líquido polar), portanto, este resultado é consistente com a teoria. 
Tabela 1 - Valores doserros relativos médios calculados pela comparação entre os dados simulados e os dados experimentais

\begin{tabular}{|c|c|c|c|}
\hline \multirow{2}{*}{$\begin{array}{c}\text { Modelo de coeficiente } \\
\text { de atividade }\end{array}$} & & \multicolumn{2}{|c|}{ Erro relativo médio [\%] } \\
\cline { 3 - 4 } & $\mathrm{n}$ & Fase líquida & Fase vapor \\
\hline UNIFAC & 15 & 16,13 & 12,24 \\
\hline NRTL & 15 & 13,49 & 12,46 \\
\hline UNIQUAC & 15 & 12,97 & 12,28 \\
\hline Wilson & 15 & 13,10 & 11,75 \\
\hline
\end{tabular}

Para a validação do ELL do ternário, primeiro é necessário fazer uma ressalva sobre a temperatura. Na simulação apresentada, o reator utilizado para a reação é do tipo conversão fixa. Em outras palavras, a temperatura do reator não interfere nas frações produzidas pela reação já que a conversão dos reagentes foi fixada no próprio equipamento em 50\% do reagente etanol. Portanto, a temperatura do reator foi alterada para $20^{\circ} \mathrm{C}$ em vez de $40^{\circ} \mathrm{C}$, temperatura original da simulação, para que fosse possível comparar os dados obtidos na simulação com os dados experimentais disponíveis para o ELL do ternário (MERZOUGUI et al., 2011). Este artigo apresenta dados experimentais de ELL para ternários de água-álcool-DEE ou DCM a $20{ }^{\circ} \mathrm{C}$. Os dados de ELL foram estimados usando o modelo NRTL e os parâmetros de interação binária foram estimados com o uso do modelo de coeficiente de atividade UNIQUAC. Para fins de comparação, apenas os modelos NRTL e UNIQUAC foram utilizados nesta etapa para calcular as linhas de amarração e a binodal.As Figuras 3 e 4 mostram os resultados obtidos.

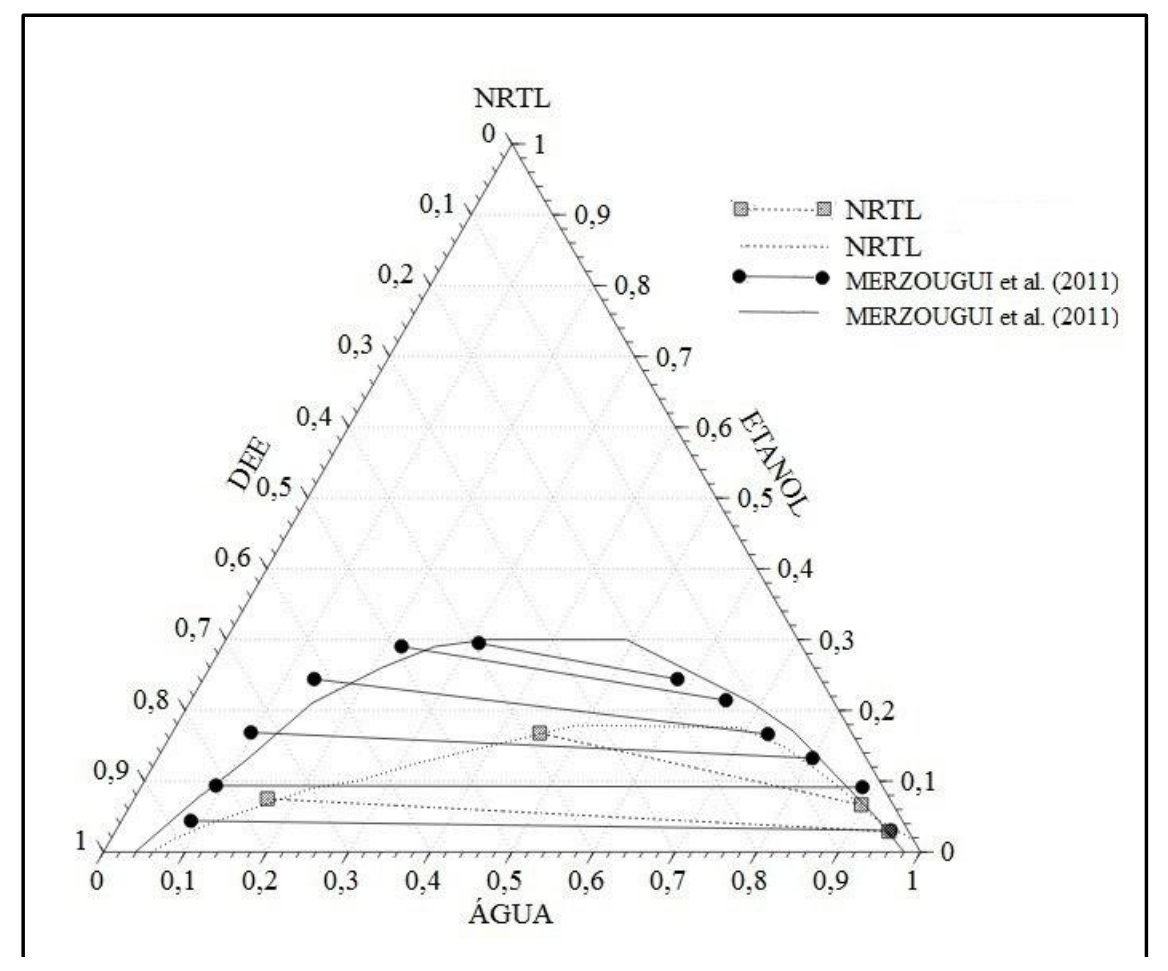

Figura 3- Diagrama ternário para água-etanol-DEE, linhas de amarração e binodal experimentais e linhas de amarração e binodal pelo NRTL

Na Figura 3, a binodal obtida pelo modelo NRTL apresenta uma região líquida bifásica menor do que os dados experimentais de MERZOUGUI et al (2011), o que explica o porquê de apenas duas linhas de amarração terem sido obtidas pelo simulador. Outra observação que pode ser feita é o fato de que as linhas de amarração calculadas pela simulação não têm a mesma inclinação que as linhas de amarração experimentais. 


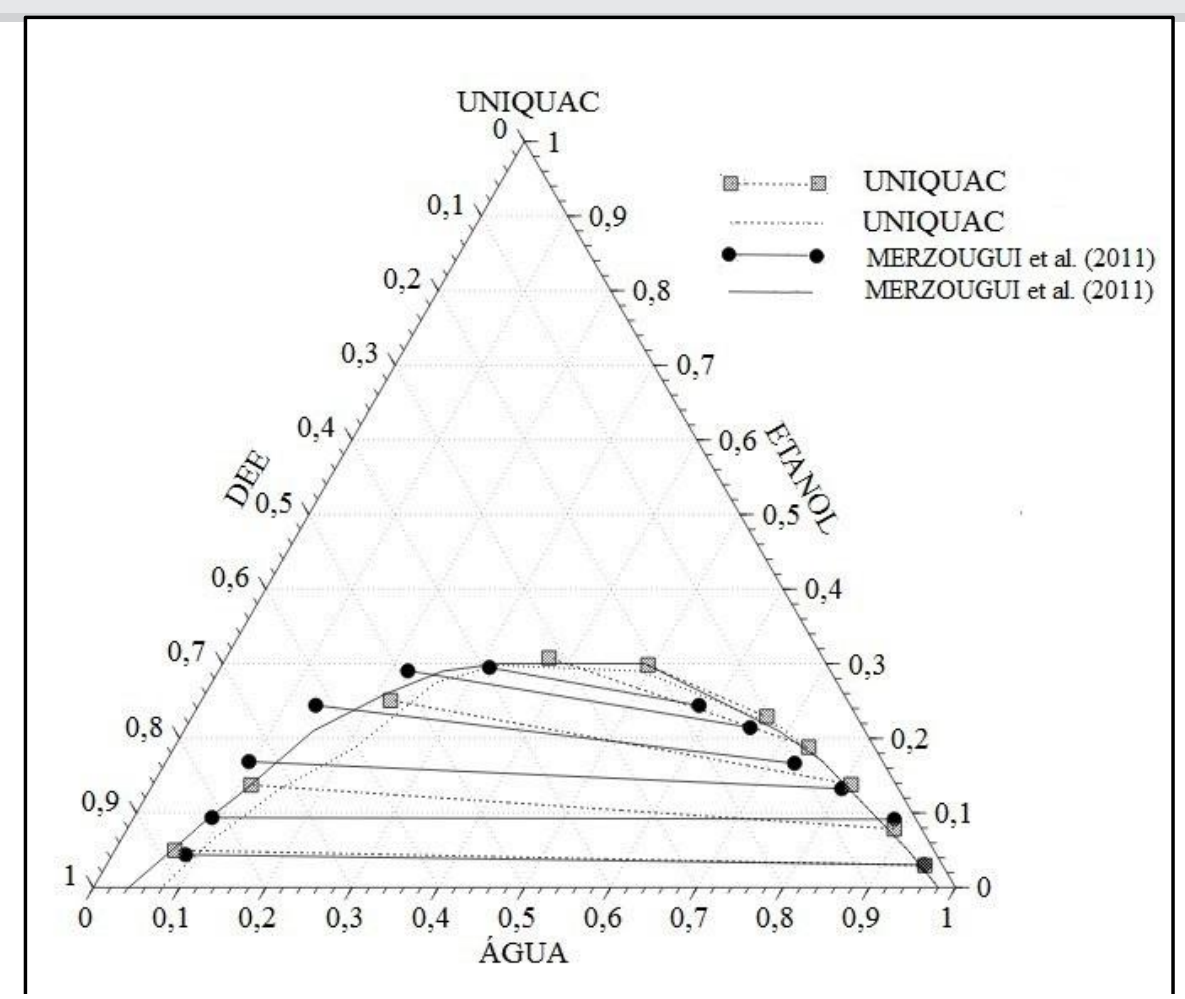

Figura 4- Diagrama ternário para água-etanol-DEE, linhas de amarração e binodalexperimentais e linhas de amarração e binodal pelo UNIQUAC

Para Figura 4, foram encontradas cinco linhas de amarração quando utilizado o modelo UNIQUAC. Todas as linhas de amarração se encontram próximas a binodal experimental e apenas algumas linhas de amarração não tem o mesmo comportamento da inclinação que as experimentais. A binodal obtida pelo modelo UNIQUAC tem o comportamento semelhante abinodal experimental, diferindo-se apenas na fase orgânica do diagrama.

É possível observar na Tabela 2 que os valores encontrados para oserros relativos médios para o modelo NRTL são, em sua maioria, superior aos valores encontrados pelo modelo UNIQUAC. O modelo UNIQUAC, tanto qualitivamentequanto numericamente, é mais adequado para descrever as linhas de amarração. De qualquer forma, o modelo UNIQUAC apresentaria os melhores resultados, já que foi com este modelo que as linhas de amarração foram estimadas.

Tabela 2- Valores doserros relativos médioscalculados pela comparaçãodas linhas de amarração experimentais e simuladas no $\mathrm{COCO}$

\begin{tabular}{|c|c|c|c|c|c|c|c|}
\hline \multirow{3}{*}{$\begin{array}{c}\text { Modelo de coeficiente de } \\
\text { atividade }\end{array}$} & \multirow{3}{*}{$\mathrm{n}$} & \multicolumn{6}{|c|}{ Erro relativo médio [\%] } \\
\hline & & \multicolumn{3}{|c|}{ Fase orgânica } & \multicolumn{3}{|c|}{ Fase aquosa } \\
\hline & & $\mathrm{X}_{\text {água }}$ & $\mathrm{X}_{\text {etanol }}$ & $\mathrm{x}_{\mathrm{DEE}}$ & $\mathrm{X}_{\text {água }}$ & $\mathrm{X}_{\text {etanol }}$ & $\mathrm{X}_{\mathrm{DEE}}$ \\
\hline NRTL & 2 & 0,72 & 15,85 & 29,04 & 243,84 & 75,12 & 32,84 \\
\hline UNIQUAC & 5 & 0,62 & 8,06 & 10,57 & 94,73 & 23,05 & 12,42 \\
\hline
\end{tabular}

A Tabela 3 apresenta os erros relativos médios para os pontos da binodal. Os erros obtidos para as frações de água em ambos os modelos são semelhantes, mas para as outras frações os valores apresentados são bastante diferentes. Considerando os resultados obtidos para a binodal, o melhor modelo é o UNIQUAC. 
Tabela 3- Valores dos erros relativos médios calculados pela comparação da binodal experimental e simuladas no COCO

\begin{tabular}{|c|c|c|c|c|}
\cline { 3 - 5 } \multicolumn{2}{c|}{} & \multicolumn{3}{c|}{ Erro relativo médio [\%] } \\
\hline Modelo de coeficiente de atividade & $\mathrm{n}$ & $\mathrm{W}_{\text {água }}$ & $\mathrm{W}_{\text {etanol }}$ & $\mathrm{W}_{\text {DEE }}$ \\
\hline NRTL & 11 & 3,87 & 37,43 & 31,74 \\
\hline UNIQUAC & 11 & 3,85 & 1,71 & 1,87 \\
\hline
\end{tabular}

A diferença entre as linhas de amarração e binodais simuladas e as linhas de amarração e binodal experimentais é explicada pelo fato de que os modelos NRTL e UNIQUAC apresentados no artigo são diferentes dos modelos utilizados no simulador de processos COCO, assim como os parâmetros de interação binária regredidos para seus respectivos modelos.

Para a comparação do ELV do ternário foi analisado duas pressões distintas: 5,08 e 7,12 atm (DDBST GmbH, 2014).Não foi encontrado na literatura dados sobre o ELV deste ternário a pressões mais baixas. Como os modelos analisados não apresentam pressão nas suas equações, pode-se considerar que a influência desta variável sobre o cálculo dos coeficientes de atividade é pequena e deste modo tais modelos foram validados a uma pressão diferente das de simulação.Os modelos analisados foram: UNIFAC, NRTL, Wilson e UNIQUAC. Para a pressão de 7,12 atm, não foi calculado pelo simulador nenhuma das temperaturas experimentais para o modelo UNIFAC, por isso não há resultados na Tabela 4. A Tabela 4 mostra os resultados obtidos para os outros modelos.Para ambas as pressões, o modelo que apresentou os menores valores para o erro relativo médio foi o UNIQUAC.

Tabela 4- Valores doserros relativos médios calculados pela comparação do ELV do ternário águaetanol-DEE

\begin{tabular}{|c|c|c|c|c|c|c|c|}
\hline & & & & & & & \\
\hline & & & & rro relat & nédio [\% & & \\
\hline & & & ase líqu & & & ase vap & \\
\hline Modelo & $\mathrm{n}$ & $\mathrm{x}_{\mathrm{DEE}}$ & $\mathrm{X}_{\text {etanol }}$ & $\mathrm{X}_{\text {água }}$ & $\mathrm{y}_{\mathrm{DEE}}$ & $\mathrm{y}_{\text {etanol }}$ & Yágua \\
\hline & & & & & & & \\
\hline UNIFAC & 8 & 142,07 & 39,75 & 34,49 & 114,86 & 47,95 & 60,42 \\
\hline NRTL & 12 & 73,32 & 34,95 & 109,82 & 163,64 & 43,43 & 41,88 \\
\hline Wilson & 11 & 57,14 & 36,20 & 124,59 & 100,48 & 44,18 & 45,29 \\
\hline UNIQUAC & 12 & 79,27 & 46,67 & 46,67 & 100,27 & 42,03 & 45,72 \\
\hline & & & & & & & \\
\hline NRTL & 9 & 92,13 & 39,68 & 126,66 & 130,82 & 38,15 & 47,87 \\
\hline Wilson & 9 & 75,72 & 38,12 & 123,40 & 134,19 & 38,56 & 47,5 \\
\hline UNIQUAC & 9 & 71,48 & 37,36 & 127,02 & 94,75 & 38,71 & 48,5 \\
\hline
\end{tabular}

Além de verificar qual modelo termodinâmico melhor representa os equilíbrios de fase, foi investigado o efeito que a termodinâmica tem sobre o processo químico simulado.Para esta análise foi selecionado um parâmetro operacional e seu comportamento foi comparado quando o modelo termodinâmico da simulação era alterado.O parâmetro operacional analisado foi a volatilidade relativa das colunas de destilaçãoChemSepUO_2 e ChemSepUO_3. Para isto, foram coletados dados do simulador ChemSep acerca a volatilidade relativa paraos modelos: UNIFAC, NRTL, Wilson e UNIQUAC. Os resultados são mostrados na Figura 5. 


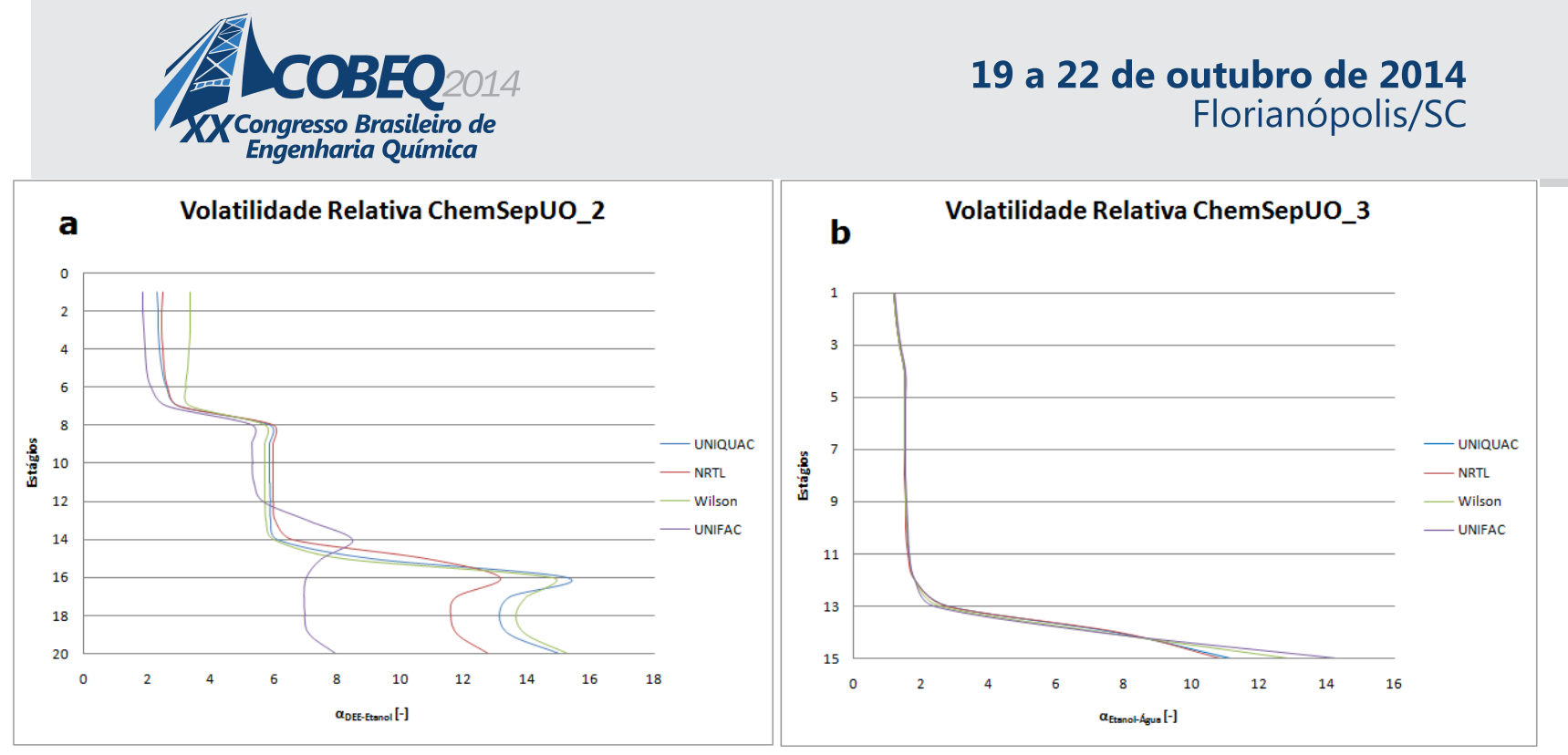

Figura 5- Volatilidade relativa água-etanol-DEE para as colunas de destilação ChemSepUO_2 e ChemSepUO_3 para os modelos termodinâmicos analisados

Para a primeira coluna, responsável pela separação do DEE do binário água-etanol, a escolha do modelo termodinâmico é bastante importante. Isto pode ser visto na Figura 5-a. Os modelos geraram uma tendência de comportamento semelhante, mas como valore de $\alpha_{\text {DEE-tanol }}$ distintos. Já a Figura 5-b, os resultados obtidos foram bastante semelhantes, se diferindo apenas entre os estágios 13 e 15.

A Tabela 5 mostra as cargas térmicas nos três equipamentos envolvidos na simulação: um reator de conversão fixa e duas colunas de destilação simples com um condensador total e reboiler parcial. Não é possível indicar o modelo com o menor custo energético até porque não existe dados operacionais publicados sobre esta planta industrial simulada para que seja feita esta comparação. Mas, é possível notar a variação das cargas térmicas dos equipamentos com a variação dos modelos termodinâmicos. Dependendo do modelo termodinâmico selecionado para a síntese de um projeto, os custos de energia podem ser bastante elevados.

Tabela 5 - Influência do modelo termodinâmico para o cálculo do coeficiente de atividade na carga térmica dos equipamentos do processo

\begin{tabular}{|c|c|c|c|c|}
\hline \multirow{2}{*}{ Equipamento } & \multicolumn{4}{|c|}{ Cargas Térmicas [W] } \\
\cline { 2 - 5 } & UNIFAC & NRTL & Wilson & UNIQUAC \\
\hline Reactor1 & -207.553 & -173.454 & -174.794 & -171.619 \\
\hline ChemSepUO_2 & $2,23.10^{6}$ & $2,71.10^{6}$ & $2,77.10^{6}$ & $2,68.10^{6}$ \\
\hline ChemSepUO_3 & $3,27.10^{6}$ & $3,29.10^{6}$ & $3,23.10^{6}$ & $3,28.10^{6}$ \\
\hline
\end{tabular}

\section{CONCLUSÕES}

A conclusão que pode ser tomada é que a escolha dos pacotes termodinâmicos podem influenciar fortemente os resultados de uma simulação. A escolha errada durante o projeto de processos químicos pode resultar em equipamentos mal projetados, que nem alcançam os objetivos desejados e consomem mais energia do que é realmente necessário. Os modelos termodinâmicos também influenciam os quantificadores termodinâmicos da facilidade/dificuldade da separação, neste trabalho representado pela volatilidade relativa, e por meio desta informação é possível determinar as regiões de uma coluna de destilação mais suscetivel a erros na simulação. Este tipo de informação é útil para avaliar o impacto de escolhas termodinâmicas na planta física visando eficiência e economia energética. 


\section{AGRADECIMENTOS}

Os autores agradecem à FAPEMIG (Processo PCE-00089-14) pelo apoio concedido.

\section{REFERÊNCIAS}

ChemSep - Modeling Separation Processes, <http://www.chemsep.com/>, acessado 03/02/2014.

COCO - the CAPE-OPEN to CAPE-OPEN simulator, < $\underline{\text { http://www.cocosimulator.org/ }>}$ Acessado 03/02/2014.

DDBST GmbH , Vapor-LiquidEquilibrium

Data, <http://www.ddbst.com/en/EED/VLE/VLE\%20Ethanol\%3BDiethyl\%20ether\%3BWater.php> acessado 27/02/2014 .

MERZOUGUI, A.; HASSEINE, A.; KAOUCHE, A. e KORICHI, M. LLE for the extraction of alcohol from aqueous solutions with diethyl ether and dichloromethane at $293.15 \mathrm{~K}$, parameter estimation using a hybrid genetic based approach. Fluid Phase Equilibria. v. 309, p. 161-167, 2011.

PERRY, R. H.; CHILTON, C. H. e KIRKPATRICK, S. D. John H. Perry’s chemical engineers' handbook, McGraw-Hill, 1963.

SADEQ, J.; DUARTE, H. A. e SERTH, R. W. Anomalous Results from Process Simulators.Chem. Eng. Education, p. 46-51, 1997.

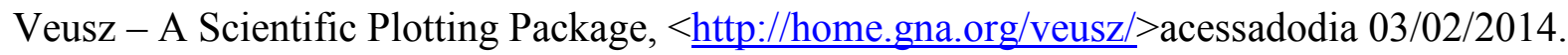

WIDAYAT; ROESYADI, A.; RACHIMOELLAH, M. Diethyl Ether Production Process with Various Catalyst Type.Internat. J. of Sci. and Eng., v.4(1), p. 6-10, 2013. 